\title{
A Two Stage DC-DC Converter with Isolation for Renewable Energy Applications
}

\author{
T. Saravanan ${ }^{1 *}$, V. Srinivasan ${ }^{2}$ and V. P. Sandiya ${ }^{3}$ \\ 'Professor \& Head, Department of ETC, Bharath University, Chennai-600073, India; saravanan.etc@bharathuniv.ac.in \\ 2PG Students, Valliammai Engineering College, Chennai; Sriniraj99@yahoo.com \\ ${ }^{3}$ Assistant Professor, Valliammai Engineering College, Chennai; ezhil.power@gmail.com
}

\begin{abstract}
In this work, the design and development of a DC-DC Converter using a two stage buck-boost operation is discussed. This converter is suitable for low power application such as a matrix LED lighting system. The advantage of this converter is that instead of using two controlled switches as in case of a conventional converter, this circuit uses one MOSFET as a switch. The circuit uses two inductors and two capacitors as energy transferring elements that are able to provide a good quality, high efficiency power supply to the load. The design of TSBB converter is analyzed with closed loop control using a PI controller circuit. Even with smaller values of inductor and capacitor a highly efficient power supply can be made. The main feature of this circuit compared to the other circuits proposed previously is that have a galvanic isolation between the load and the source is provided. Thus, by a suitable controller and galvanic isolation, the ripple magnitude in the output is reduced considerably. A simulation model of the TSBB converter with results is presented.
\end{abstract}

Keywords: Two Stage Buck-boost (TSBB), LED Matrix, Duty Cycle ( $\alpha$ ), Galvanic Isolation, Discontinuous Conduction Mode (DCM), Continuous Conduction Mode (CCM)

\section{Introduction}

Energy efficient lighting is the need of the day and is becoming an area of continuous research. Illumination engineers and architects require an efficient and aesthetic lighting. In this way, the LED lighting system using Matrix type of LED arrangement is becoming more popular. But the problem with such type of lighting is the power supply. Even the recent types of power supplies used for led illumination system are poor in efficiency and they are unreliable. The tungsten lamps provide only $8-10 \mathrm{~lm} / \mathrm{W}$ and very inefficient, they are then replaced by fluorescent lamps. Now with the efficacies of an LED is as high as 100 $\mathrm{lm} / \mathrm{W}$ [1] they are mostly preferred. The problem with the LED lighting is that they cannot be supplied directly from the DC or voltage. Therefore there should be some kind a device to regulate the power fed to the lighting system all these mean a power supply should be developed to act as a driver circuit to the LED matrix lamp[2]. With the double buck boost converter, two converters are cascaded with a single switch and it includes two low value inductors and capacitors and suitably placed diodes thus the LED lamp can be supplied with low current low ripple and high efficiency power. In Section II, the working of TSBB converter [3] is presented. In Section III, the analysis and design of the TSBB converter [4] in various modes is detailed. In Section IV, Simulation results of TSBB converter is discussed. Section V discusses on the laboratory prototype made and in the section VI results and conclusions are discussed.

*Corresponding author:

T. Saravanan (pci_saravanan@yahoo.cp.in) 


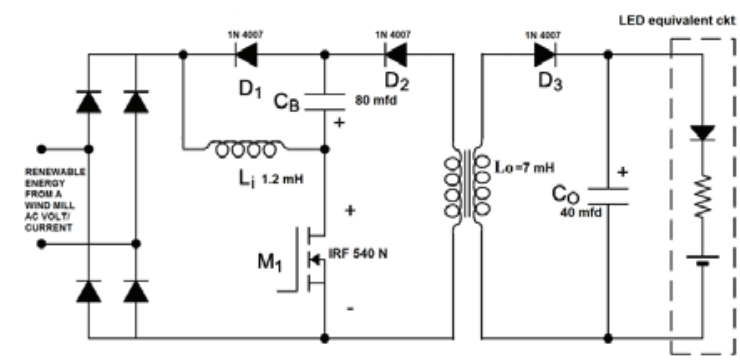

Figure 1. Schematic circuit of a TSBB converter with isolation.

\section{Two Stage Buck Boost Converter}

Figure 1 shows the schematic circuit of the TSBB converter. The converter acts as two buck-boost converters which are electrically isolated and connected to the load. The input buck-boost converter is made up by Li, D1, CB, and M1, and the output buck-boost converter here acts as a fly wheel converter due to addition of galvanic isolation and comprises of LO, D3, CO. The first converter produces a reverse output across the capacitor CB which is corrected by the second converter and a positive output voltage with respect to ground, thereby simplifying the measurement of the load current, in the closed-loop operation, also reducing the sensing circuitry and hence the cost.

By operating the input inductor $\mathrm{Li}$ in discontinuous conduction mode (DCM), the average current through the line will be almost in phase with the line voltage, which results in a line power factor near-unity, this is important if the converter operates from mains voltage in a grid. Also, the output inductance LO can be operated either in continuous conduction mode (CCM) or Discontinuous Conduction Mode. DCM results in a bus voltage across $\mathrm{CB}$ that is independent of duty cycle and output power. But there is a drawback in above said DCM since it requires a higher value of capacitance to be connected across the output in order to achieve reduced current ripple in the output of the TSBB converter. So to avoid high current ripple in the output and to reduce the value of the output capacitance, CCM operation is preferred. In addition to above, to reduce the ripple voltage occurring at low frequencies, the second stage of the TSBB is operated with $\alpha=50 \%$ (duty cycle), since the duty cycle is multiplied by the buck-boost converter voltage ratio. In this way, it will be possible even to use a film capacitor to implement the output capacitance; this gives the TSBB converter longer life rating and improved efficiency over the use of electrolytic capacitors.
Moreover, with a careful design of the converter, the bus capacitor can also be made low enough to be implemented with film technology, thus avoiding the low-life-rating electrolytic capacitors in the whole converter. This implies the design of the converter so that it operates with a a lower than 0.5 . In this manner, the output converter voltage ratio will be lower than one, thus reducing the low-frequency voltage ripple in the same amount.

The equivalent circuits for the operation of the TSBB converter for one cycle a switching period are shown in Figure 2.

\subsection{Need for Isolation}

Among the regulators non-isolated switching regulators are very common, or Point of Load (POL) converters. Although low cost and simple, these converters suffer from one disadvantage in that there is an electrical connection between the input and output. Many safety agency bodies require a separation from the applied input voltage and the output voltage which is often user accessible. An isolated DC-DC converter will have a high frequency transformer providing that barrier. This barrier can withstand anything from a few hundred volts to several thousand volts, as is required for medical application. A second advantage of an isolated converter is that the output can be configured to be either positive or negative. Where many users get confused concerns how to connect the input up, particularly with the differences between a data com system where the input negative is connected to chassis and a telecom system input positive is connected to chassis.

\section{Analysis of TSBB Converter}

In this section, the TSBB converter is analyzed when operated from the voltage supplied by a renewable energy source namely a windmill for, achieving a low-ripple current through the power-LED load. For the sake of the analysis it is assumed that the line voltage is a perfectly sinusoidal whereas in case of a real wind mill the output sine wave may have voltage sag and swell. The input voltage is thus given as

$$
\mathrm{vp}(\mathrm{t})=\mathrm{Vm} \sin \omega \mathrm{t} .
$$

\subsection{Line Current}

The input current ip corresponds to the current through the inductance Li during the time interval $0-\alpha \mathrm{TS}$, where $\alpha$ is the transistor duty cycle and TS is the transistor switching 
period. This current is modulated by the rectified line voltage [4], as. Thus, the value of the input current averaged at line frequency can be calculated as follows

$$
\left\langle i_{p}\right\rangle=\frac{\alpha^{2} V p}{2 L_{i} f_{s}} \sin \omega t
$$

where $i_{\text {p_peak }}$ is the instantaneous peak current in each switching period, $\mathrm{f}_{\mathrm{s}}$ is the switching frequency, $\mathrm{Vp}$ is the peak line voltage, and $\omega$ is the line angular frequency. The mean input power $\mathrm{P}_{\mathrm{i}}$ can now be calculated as [5]

$$
P_{i}=\frac{\alpha^{2} V_{p}^{2}}{4 L_{i} f_{S}}
$$

\subsection{Output and Bus Voltages}

The output voltage $\mathrm{V}_{\mathrm{O}}$ for the ideal converter can be obtained by equalling input and output powers. The output power is obtained as follows:

$$
\mathrm{P}_{\mathrm{o}}=\frac{\mathrm{V}_{\mathrm{o}}^{2}}{\mathrm{R}}
$$

with $\mathrm{R}$ being the static equivalent resistance[5] of the LED load, which can be obtained by the ratio between the $\mathrm{dc}$ values of LED voltage $\left(\mathrm{V}_{\mathrm{LED}}\right)$ and current $\left(\mathrm{I}_{\mathrm{LED}}\right)$ at each operating point

$$
\mathrm{R}=\frac{\mathrm{V}_{\gamma}}{\mathrm{I}_{\mathrm{LED}}}+\mathrm{R}_{\gamma}
$$

Where $\mathrm{V}_{\gamma}$ and $\mathrm{R}_{\gamma}$ are the voltage and resistance parameters of the LED lamp equivalent circuit [6] Then, assuming $100 \%$ efficiency, by equalling (2) and (3), the output voltage is finally obtained

$$
\mathrm{V}_{\mathrm{o}}=\frac{\alpha \mathrm{V}_{\mathrm{p}}}{2 \sqrt{\mathrm{D}}}
$$

where $\mathrm{D}$ is a non-dimensional factor given by

$$
\mathrm{D}=\frac{\mathrm{f}_{\mathrm{s}} \mathrm{L}_{\mathrm{i}}}{\mathrm{R}}
$$

Since the output stage corresponds to a buck-boost converter (flywheel converter) operating in CCM, the bus voltage $\mathrm{V}_{\mathrm{B}}$ can be calculated by using the voltage conversion ratio for this converter

$$
\mathrm{V}_{\mathrm{B}}=\frac{1-\alpha}{\alpha} \mathrm{V}_{\mathrm{o}}=\frac{(1-\alpha) \mathrm{V}_{\mathrm{g}}}{2 \sqrt{\mathrm{D}}}
$$

As can be seen from (5) and (7), when operating the input stage in DCM and the output stage in CCM, the bus and output voltages are reversely dependent on the duty cycle $\alpha$.

For example, if the a increases, the output voltage increases, and the bus voltage decreases in the same amount. The sum of both voltages does not depend on the duty cycle, being only proportional to the line peak voltage, as given by the following:

$$
\mathrm{V}_{\mathrm{B}}+\mathrm{V}_{\mathrm{o}}=\frac{\mathrm{V}_{\mathrm{g}}}{2 \sqrt{\mathrm{D}}}
$$

It must be noted that the input stage must be operated in DCM under any load the limit duty cycle alimit can be obtained from the voltage conversion ratio in the DCM-CCM boundary

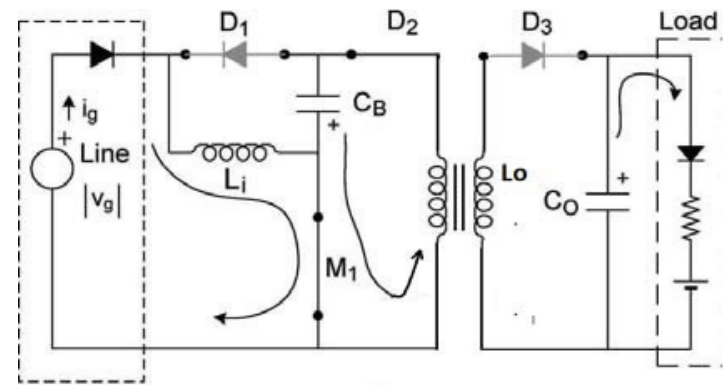

(a)

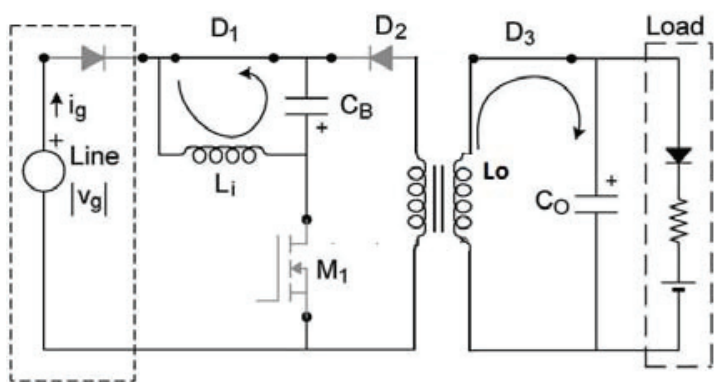

(b)

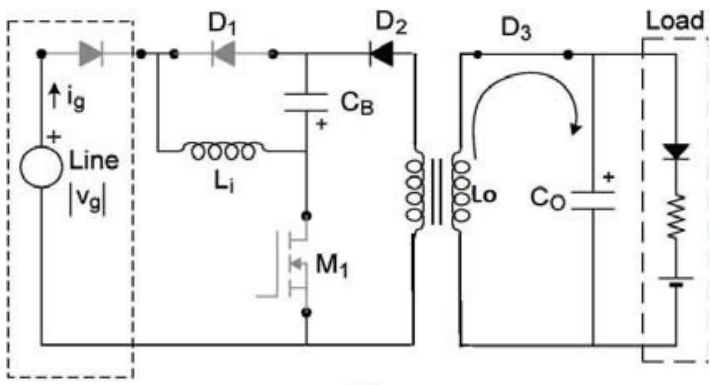

(c)

Figure 2. Equivalent circuits for the operation of the TSBB converter. (a) Interval I: $0<t<D T S$. (b) Interval II: DTS $<t<$ $D T S+t 1$. (c) Interval III: DTS $+t 1<t<T S$. 


$$
\alpha_{\text {limit }}=\frac{1}{1+\mathrm{V}_{\mathrm{p}} / \mathrm{V}_{\mathrm{B}}}
$$

Till the actual duty cycle is lower than the limit value given by (9), the first stage will operate in DCM mode only.

\subsection{Energy Transferring Components}

The value of the energy transferring element at the input which is $L_{i}$ is calculated based on the output power and assuming $100 \%$ efficiency the value of inductance is so obtained to be

$$
L_{i}=\frac{\alpha^{2} V_{p}^{2}}{4 P_{o} f_{s}}
$$

Similarly the value of the capacitor which is another energy transferring element called as bus capacitor $\mathrm{CB}$ is calculated so as to limit the low-frequency ripple of the bus voltage, this bus voltage is what applied to the second stage of the TSBB converter. The current through this capacitor is also the currents through diodes D1 and D2. In these diodes, only the current through D1 is modulated by a rectified line frequency. In order to calculate the bus ripple, the low-frequency component of the current through D1 must be obtained. The average current through D1 can be calculated as follows:

$$
\left\langle\mathrm{i}_{\mathrm{D} 1}\right\rangle=\frac{1}{\mathrm{~T}_{\mathrm{s}}} \frac{\mathrm{i}_{\text {D1_peak }} \mathrm{t}_{1}}{2}
$$

Where $i_{\text {D1_peak }}$ is the peak current through D1 in each switching period and $t_{1}$ is the time needed by this current to reach zero. Both values are changing after double the line frequency and have the following values:

$$
\begin{gathered}
\mathrm{i}_{\text {D1_peak }}=\frac{\mathrm{V}_{\mathrm{p}}}{\mathrm{L}_{\mathrm{i}}} \alpha \mathrm{T}_{\mathrm{s}} \\
\mathrm{t}_{1}=\frac{\alpha \mathrm{V}_{\mathrm{p}} \mathrm{T}_{\mathrm{s}}}{\mathrm{V}_{\mathrm{B}}}
\end{gathered}
$$

Using (12) and (13) in (11), the D1 average current is given as follows

$$
\left\langle\mathrm{i}_{\mathrm{D} 1}\right\rangle=\frac{\alpha^{2}}{2 \mathrm{~V}_{\mathrm{B}} \mathrm{f}_{\mathrm{s}} \alpha \mathrm{T}_{\mathrm{s}} 2 \mathrm{~L}_{\mathrm{i}}} \mathrm{L}_{\mathrm{i}} 2 \mathrm{i}_{\text {D1_peak }}{ }^{2}
$$

Equation (14) can also be expressed as follows:

$$
\left\langle\mathrm{i}_{\mathrm{D} 1}\right\rangle=\frac{\alpha^{2} \mathrm{~V}_{\mathrm{p}}^{2}}{2 \mathrm{~V}_{\mathrm{B}} \mathrm{f}_{\mathrm{s}} \mathrm{L}_{\mathrm{i}}}
$$

Then, the low-frequency ac component circulating through $\mathrm{D} 1$ and $\mathrm{C}_{\mathrm{B}}$ is given by

$$
\left\langle\mathrm{i}_{\mathrm{D} 1}\right\rangle_{\mathrm{ac}}=\frac{\alpha^{2} \mathrm{~V}_{\mathrm{p}}^{2}}{4 \mathrm{~V}_{\mathrm{B}} \mathrm{f}_{\mathrm{s}} \mathrm{L}_{\mathrm{i}}}
$$

Now, the low-frequency peak-to-peak ripple voltage across capacitor $\mathrm{C}_{\mathrm{B}}, \Delta \mathrm{V}_{\mathrm{B} \_\mathrm{LF}}$ can be obtained as

$$
\Delta \mathrm{V}_{\mathrm{B} \_\mathrm{LF}}=\frac{\alpha^{2} \mathrm{~V}_{\mathrm{p}}^{2}}{8 \pi \mathrm{V}_{\mathrm{B}} \mathrm{L}_{\mathrm{i}} \mathrm{C}_{\mathrm{B}} \mathrm{f}_{\mathrm{s}} \mathrm{f}_{\mathrm{L}}}
$$

current through diode D1, obtained from (16), and $\mathrm{f}_{\mathrm{L}}$ is the line frequency. The necessary bus capacitance for a given peak-to-peak ripple in the bus voltage is then calculated from (17) as follows:

$$
\mathrm{C}_{\mathrm{B}}=\frac{\alpha^{2} \mathrm{~V}_{\mathrm{g}}^{2}}{8 \pi \mathrm{V}_{\mathrm{B}} \mathrm{L}_{\mathrm{i}} \Delta \mathrm{V}_{\mathrm{B}_{\mathrm{LF}} \mathrm{f}} \mathrm{f}_{\mathrm{S}} \mathrm{f}_{\mathrm{L}}}
$$

Note that, as long as the output buck-boost converter operates in CCM, the LED current ripple at low frequency depends only on the bus voltage ripple and, thus, on the bus capacitance $\mathrm{C}_{\mathrm{B}}$. The output capacitance $\mathrm{C}_{\mathrm{O}}$ has no effect on this low frequency ripple. Finally, the output inductance and capacitance $\mathrm{L}_{\mathrm{O}}$ and $\mathrm{C}_{\mathrm{O}}$ are obtained using the wellknown expressions for a buck-boost converter operating in $\mathrm{CCM}$

$$
\begin{gathered}
\mathrm{L}_{\mathrm{o}}=\frac{\alpha \mathrm{V}_{\mathrm{B}}}{0.5 \Delta \mathrm{I}_{\mathrm{Lo} \_H F_{\mathrm{HF}}} \mathrm{f}_{\mathrm{s}}} \\
\mathrm{C}_{\mathrm{o}}=\frac{\alpha \mathrm{I}_{\mathrm{o}}}{\Delta \mathrm{V}_{\mathrm{o}_{-} \mathrm{HF}} \mathrm{f}_{\mathrm{s}}}
\end{gathered}
$$

where $\Delta \mathrm{I}_{\text {Lo_HF }}$ is the $\mathrm{L}_{\mathrm{O}}$ high-frequency peak-to-peak current ripple, $\Delta \mathrm{V}_{\mathrm{O}-\mathrm{HF}}$ is the high-frequency peak-to-peak output voltage ripple, and $\mathrm{I}_{\mathrm{O}}$ is the dc current through the LED load.

\section{Simulation Results}

Simulation of the TSBB converter was done using $\mathrm{P}_{\text {Sim }}$ Software. It was found that $\mathrm{P}_{\text {sim }}$ was easier to use and the results can be obtained quicker as the time taken for the simulation was less.

The circuit configuration shown in Figure 3 shows, TSBB converter in closed loop mode using a PI controller. The values of the PI controller elements are so chosen to give a reduced steady state error. Instead of going in for 
standardised tuning methods, it was found that manual tuning gave satisfactory results. So the tuning of the PI controller was done based on trial and error method manually.

The Proportional gain (Kp) is given by the equation (21) where the values of $R_{1}$ and $R_{2}$ are determined by manual tuning method

$$
\mathrm{Kp}=\frac{\mathrm{R}_{2}}{\mathrm{R}_{1}}
$$

Similarly, the integral gain (Ki) is given by the equation (22) where the values of $R_{1}$ and $R_{2}$ are determined by manual tuning method [7]

$$
\mathrm{Ki}=\frac{1}{\mathrm{R}_{2} \mathrm{C}}
$$

Figure 4 shows the pulse width modulated gate signals applied to the gate source terminals of the mosfet switch.
The frequency of the gate signal is maintained at $5 \mathrm{kHz}$ with an a greater than $50 \%$. It can be noted from the output taken across the load as shown in Figure 5, which is the equivalent circuit of LED matrix, that when isolation is provided, there is drastic drop in the magnitude of voltage ripple a value of less than 3 Volts. Thus this converter provides a good regulation of output voltage and is suitable for $\mathrm{dc}$ load application involving either renewable energy or from mains power supply.

Thus the two stage buck-boost converter was simulated using $\mathrm{P}_{\text {sim }}$ software and the results were obtained as per the requirement. The output was high compared with conventional buck-boost convereters with 2 switches [8].

\section{Laboratory Setup}

A laboratory prototype was setup using electronic components on bread board and through the use of regulated power supplies, function generator and a $30 \mathrm{MHz}$

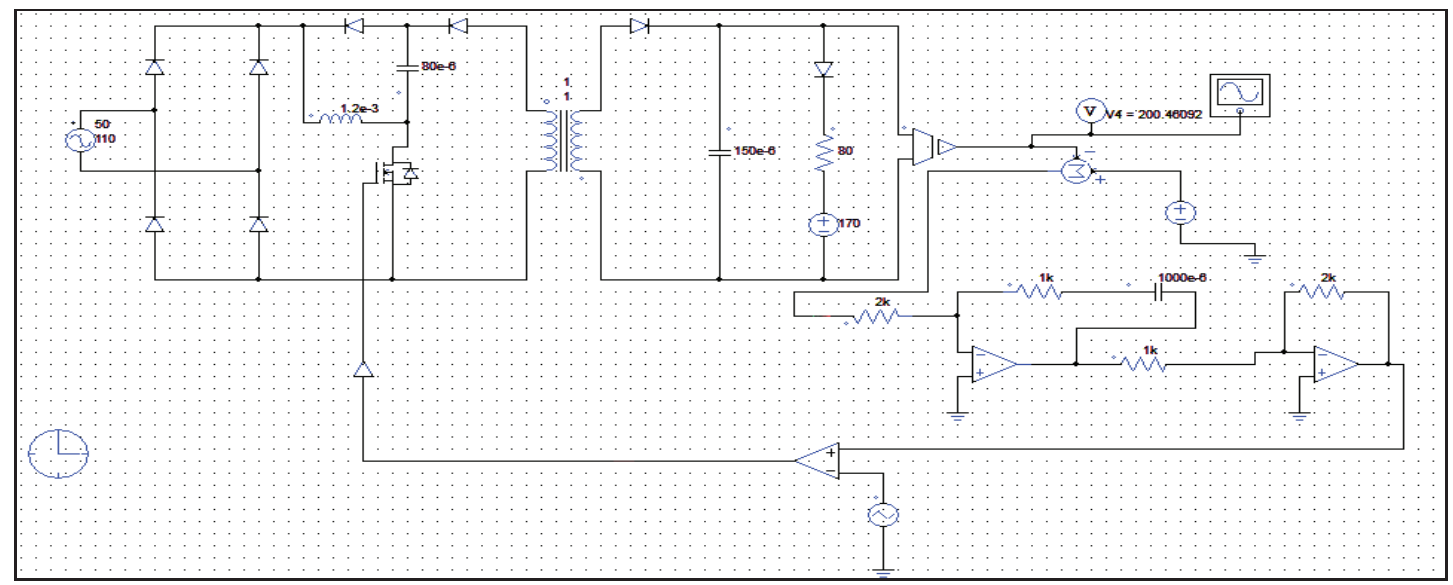

Figure 3. Simulation circuit of the DBB converter in closed loop.

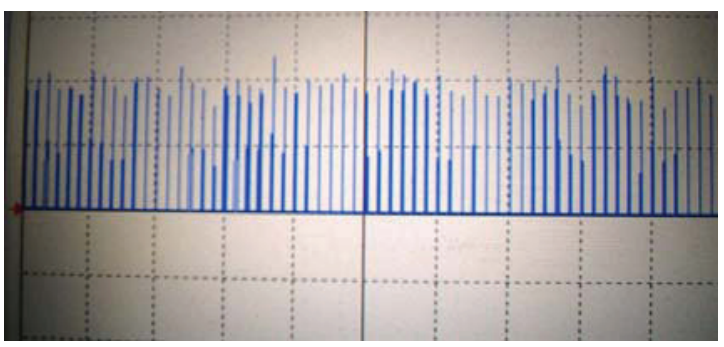

Figure 4. Screen shot of gate pulses applied to gate-source terminal of the MOSFET switch.

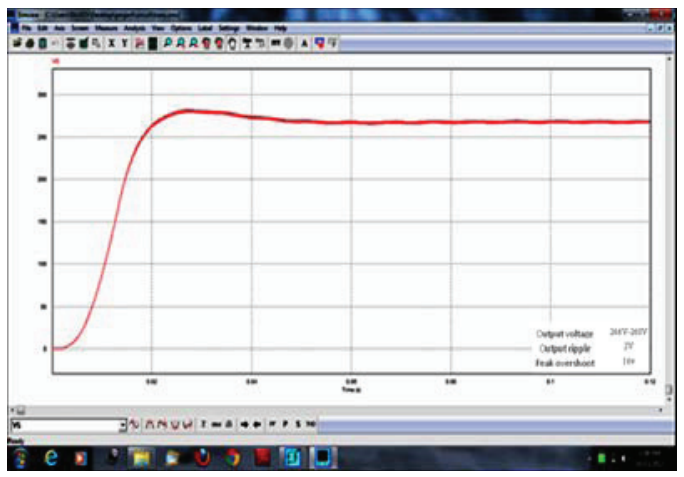

Figure 5. Simulation circuit for output voltage with galvanic isolation. 
oscilloscope. The load was a low power LED matrix lamp [3] of $20 \mathrm{~V}$ operating voltage and 36 Watts power.

The entire laboratory setup is shown in Figure 6 connected to various sources and sinks. In Figure 7, the DBB converter assembled on a bread board can be seen. The inductors used in this work is hand wound inductors as per the design given below
Gauge of copper wire used
: 32 SWG
Diameter of the copper wire
$: 0.274 \mathrm{~mm}$
Ratings of Inductors
$\mathrm{Li}=1.2$ milli Henry
Lo $=7.0$ milli Henry
N2 $=163$ turns
$\mathrm{N} 1=47$ turns

As discussed in the equations the value of the bus capacitor $\mathrm{CB}$ and output capacitor Co are of the values mentioned below

$$
\mathrm{CB}=80 \mu \mathrm{F} \mathrm{Co}=40 \mu \mathrm{F}
$$

Figure 8 shows the control section of the TSBB converter assembled on a bread board. It mainly consists of a PI controller which is manually tuned as per the values shown in the Figure 3. The output of the PI controller which is negative is given to the inverter, the output of the inverter a, DC line is now compared with high frequency triangular carrier wave to produce PWM pulses (seen in Figure 9) which is applied to the MOSFET switch.The output voltage which is a ripple free, boosted and controlled DC is shown in the $\mathrm{CRO}$ as depicted in the Figure 9. The CRO is functioning in dual channel mode with one channel showing the output voltage and the other channel showing the Pulses applied to the gate source terminals of the MOSFET. Figure 10. shows the output voltage and the reference voltage. The reference value of $20 \mathrm{~V}$ is set up using a Regulated power supply. The output across the load is measured using a multi-meter to read a value of $19.9 \mathrm{~V}$ as same as that of the reference. Thus the output voltage obtained is controlled and as per the reference.

The output which is applied to the LED matrix lamp makes it to glow brightly as shown in Figure 11. Thus the working of prototype TSBB converter with LED lamp load was successfully implemented.

\section{Conclusion}

A two stage buck boost converter (TSBB) has been successfully simulated in this paper there by a circuit configuration

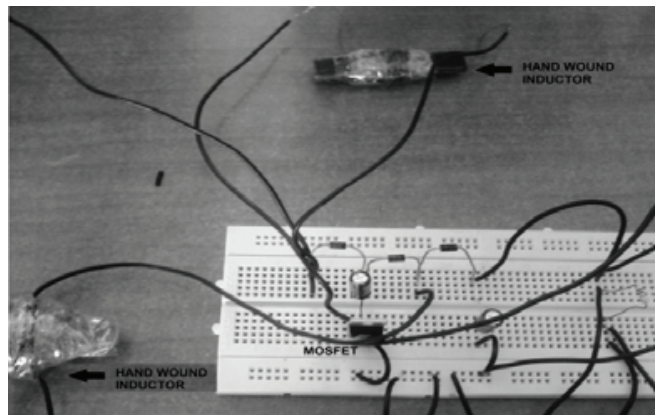

Figure 7. DBB converter on bread board with hand wound inductors.

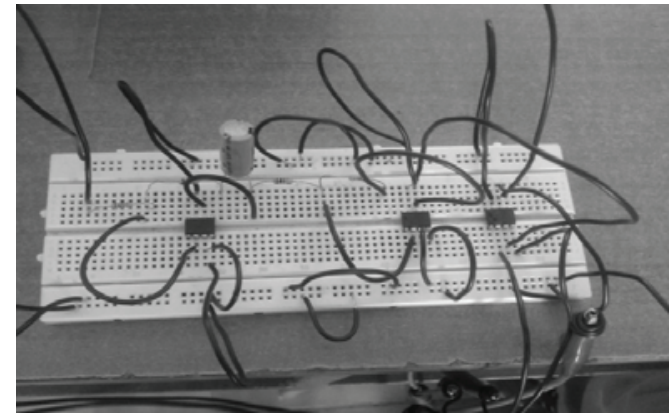

Figure 8. PI controller with inverter and PWM generator.

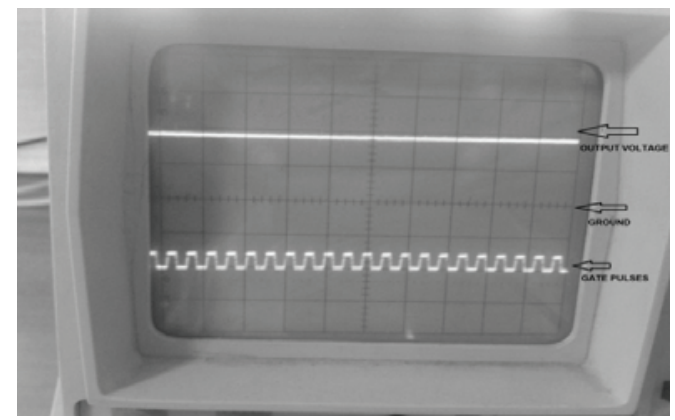

Figure 9. Gate pulses and the output voltage on the CRO.

Figure 6. Complete laboratory prototype setup. 


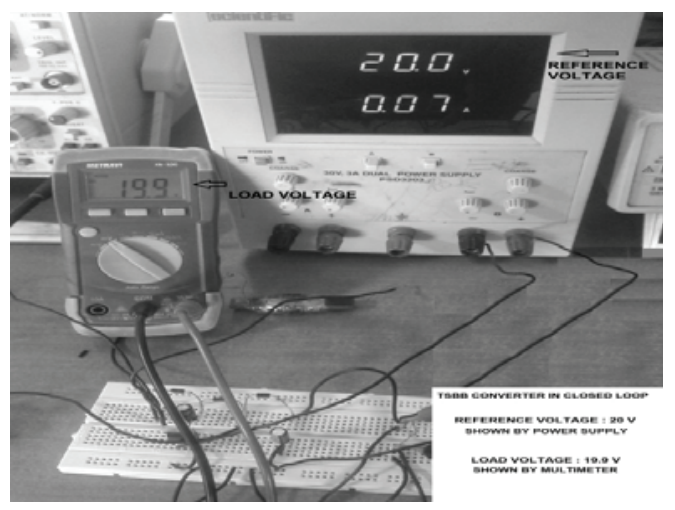

Figure 10. Reference voltage and the output voltage displayed.

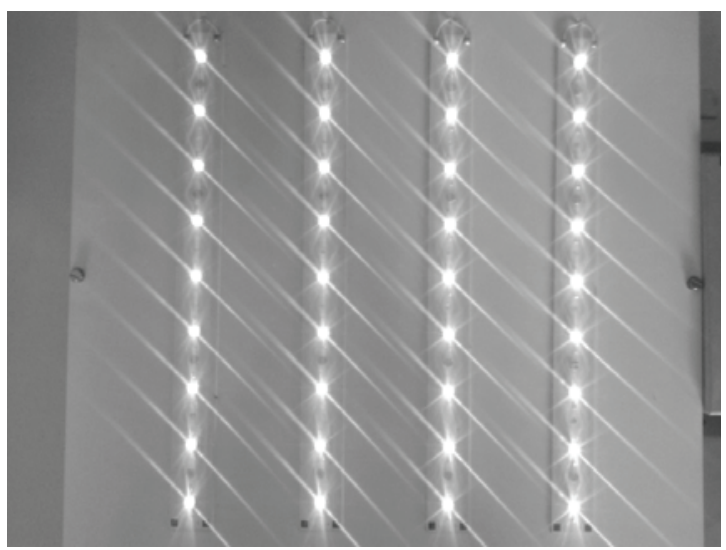

Figure 11. $36 \mathrm{~W}$ LED Matrix working from TSBB converter.

is discussed for providing quality power supply for low power applications In closed loop mode suitable controller namely the PI controller was used.

It is evident from the results discussed in the previous sections that the configurations of TSBB converter in closed loop is efficient compared to the converters using more than one control switch [9].

The TSBB converter was implemented as a laboratory prototype using electronic components on a bread board as discussed in the previous section. It can be seen that the ripple content in the output is nil and the output voltage follows the reference voltage due to the use of PI controller.

Thus realization of a buck-boost converter using a single switch was done. The output is controlled.

The added advantage of this converter is that this is suitable for power converters used for medical applications where isolation is essential. Though Isolation may end up in increase of cost, it is a safety aspect that cannot be neglected in certain applications. Thus, the configuration of a TSBB converter in closed loop mode is much efficient, safe and reliable for any low power loads.

\section{References}

1. Alonso J M, Viña J et al. (2012). Analysis and design of the integrated double buck-boost converter as a high power factor driver for power led lamps, IEEE Transactions on Industrial Electronics, vol 59(4), 1689-1697.

2. Schubert E F (2006). Light-emitting diodes, 2nd Edn., Chapter 7, Cambridge, U.K.: Cambridge Univ. Press, 201-222.

3. Cree X Lamp XP-C LEDs, 2010, Data Sheet No. CLD-DS19, Rev Chapter 6, 7-9.

4. Fang Y, Wong $\mathrm{S}-\mathrm{H}$ et al. (2009). A power converter with pulse-level-modulation control for driving high brightness LEDs, 24th Annual IEEE Applied Power Electronics Conference and Exposition, 577-581.

5. Nuttall R, Shuttleworth R et al. (2008). Design of a LED street lighting system, 4th IET Conference on Power Electronics, Machines and Drives, 436-440.

6. Yuequan H, and Jovanovic M M (2008). A novel LED driver with adaptive drive voltage, Twenty-Third Annual IEEE Applied Power Electronics Conference and Exposition, 565571.

7. Lo Y-K, Wu K-H et al. (2009). Design and implementation of RGB LED drivers for LCD backlight modules, IEEE Transactions on Industrial Electronics, vol 56(12), 4862-4871.

8. Morkoç H (2010). Handbook of nitride semiconductors and devices, Wiley-VCH Verlag GmbH \& Co. KGaA, Weinheim, vol 3.

9. Amala J N, Rajan S E et al. (2011). Design and analysis of high frequency soft-switching boost converter employing electronic PI-controller, International Conference on Emerging Trends in Electrical and Computer Technology (ICETECT), 1-132. 